\title{
Changes of Temporal Processing and Hearing in Noise after Use of a Monoaural Hearing Aid in Patients with Sensorineural Hearing Loss: A Preliminary Study
}

\author{
Yehree Kim¹, Chan Joo Yang ${ }^{2}$, Myung Hoon Yoo ${ }^{3}$, Chan II Song ${ }^{4}$, and Jong Woo Chung ${ }^{1}$ \\ ${ }^{1}$ Department of Otorhinolaryngology-Head and Neck Surgery, Asan Medical Center, University of Ulsan College of Medicine, Seoul, Korea \\ ${ }^{2}$ Department of Otolaryngology, Hanil General Hospital, Seoul, Korea \\ ${ }^{3}$ Department of Otorhinolaryngology-Head and Neck Surgery, College of Medicine, Kyungpook National University, Daegu, Korea \\ ${ }^{4}$ Department of Otorhinolaryngology, Yonsei University College of Medicine, Seoul, Korea
}

\author{
Received January 28, 2021 \\ Revised May 21, 2021 \\ Accepted June 21, 2021
}

Background and Objectives: The relationship between hearing aid (HA) use and improvement in cognitive function is not fully known. This study aimed to determine whether HAs could recover temporal resolution or hearing in noise functions. Materials and Methods: We designed a prospective study with two groups: HA users and controls. Patients older than 45 years, with a pure tone average threshold of worse than $40 \mathrm{~dB}$ and a speech discrimination score better than $60 \%$ in both ears were eligible. Central auditory processing tests and hearing in noise tests (HINTs) were evaluated at the beginning of the study and $1,3,6$, and 12 months after the use of a monaural HA in the HA group compared to the control group. The changes in the evaluation parameters were statistically analyzed using the linear mixed model. Results: A total of 26 participants (13 in the HA and 13 in the control group) were included in this study. The frequency $(p<0.01)$ and duration test $(p=0.02)$ scores showed significant improvements in the HA group after 1 year, while the HINT scores showed no significant change. Conclusions: After using an HA for one year, patients performed better on temporal resolution tests. No improvement was documented with regard to hearing in noise.

J Audiol Otol 2021;25(3):146-151

E-mail jwchung@amc.seoul.kr

KEY WORDS: Hearing aid · Cognitive function · Temporal processing · Hearing in noise.

\section{Introduction}

Hearing loss is a general problem in aged people. World Health Organization estimated that approximately one-third of persons over 65 years are affected by disabling hearing loss. The prevalence of hearing loss among people of advanced economies aged $>65$ years is $0.6 \%$, which is comparable to data from South Korea $(0.46 \%-0.5 \%)$ [1]. Globally, the prevalence of hearing impairment $\geq 35 \mathrm{~dB}$ HL for males aged $\geq 15$ years was $12.2 \%(9.7 \%-16.2 \%)$, whereas for females aged $\geq 15$ years it was $9.8 \%(7.7 \%-13.2 \%)[2]$.

Hearing loss is known to be a risk factor for cognition disorders and dementia [3]. According to the Baltimore study,

This is an Open Access article distributed under the terms of the Creative Commons Attribution Non-Commercial License (https://creativecommons.org/licenses/by-nc/4.0/) which permits unrestricted non-commercial use, distribution, and reproduction in any medium, provided the original work is properly cited. the population with hearing loss has a higher risk for having a cognitive disorder (3.4 times) than the population without hearing loss (hearing level better than $25 \mathrm{~dB}$ ) [4]. Also, hearing aid (HA) users show a lower risk for developing a cognitive disorder than hearing loss patients that do not use HAs [5]. However, among the patients with moderate sensorineural hearing loss, only $40 \%$ of them use HAs [6].

One of the hearing-related cognition abilities is central auditory processing (CAP). CAP is an ability to separate and integrate the words heard in both ears [7]. Using CAP, we can hear the fine structures of speech [8]. The ability to distinguish words in the presence of noise is an essential function to hear well and to understand everyday conversation [9]. CAP and the function of hearing in noise are important for better hearing and conversation. Understanding sound in the presence of ambient noise is an innate ability to hear. However, many patients with hearing loss suffer from decreases in this 
function [10].

Although the relationship between hearing loss and cognitive dysfunction has been suggested, a causal relation has not yet been addressed. The hearing loss itself may be related to cognitive dysfunction $[11,12]$ or hearing loss-related depression, or social segregation may lead to cognitive dysfunction [13].

The relationship between HA use and the improvement of cognitive function has previously been suggested [5]. The authors used cognitive test parameters, such as reaction time, pairs matching, and fluid intelligence to test whether the association between hearing impairment and cognition may be mediated by HA use, social isolation and/or depression. However, this relationship needs to be supported by further studies. In this study, we enrolled patients with moderate hearing loss and evaluated CAP and the hearing in noise function in these patients according to the use of a HA. Temporal resolution tests were used to evaluate CAP and hearing in noise tests (HINTs) were used to evaluate the hearing in noise function. The aim of this study was to evaluate whether HAs could recover temporal resolution or the hearing in noise function.

\section{Materials and Methods}

\section{Patients}

We designed a nonrandomized prospective study of two groups: HA users and control patients. Among patients who visited the out-patient clinic complaining of hearing loss, those older than 45 years of age, with a pure tone average (PTA) threshold worse than $40 \mathrm{~dB}$ and a speech discrimination score (SDS) better than $60 \%$ in both ears were selected to participate. We recommended the use of HAs to these patients; the actual decision to use a HA was made by the patient according to his or her preference. According to the patient's decision, participants were divided into two groups: a HA group of patients who started to use a monaural HA for the first time and a control group of patients who did not use HAs until the end of the study. HAs were used unilaterally. These patients used unilateral HAs until the end of this study (Fig. 1).

\section{Evaluation parameters}

For the selection of participants, pure tone audiometry, speech audiometry, impedance audiometry, distortion product otoacoustic emission, and auditory brainstem response were used. HAs were adjusted according to the pure tone audiometry, real ear measurement and functional gain tests. For the evaluation of CAP, temporal resolution tests (duration and frequency tests) and dichotic spondee words test were used [14-16]. For frequency test, 3 pure tones ranging from $880 \mathrm{~Hz}$ to $1,122 \mathrm{~Hz}$ are generated so that the frequency of 1 tone is

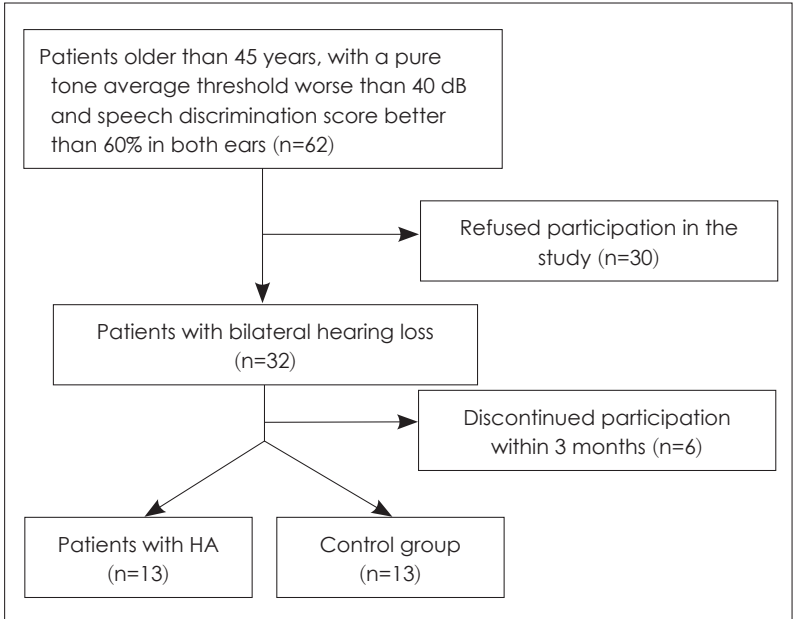

Fig. 1. Flowchart describing patients in the study. HA: hearing aid.

different from the other 2. Using these tones, various tonal combinations are created. Subject is asked to identify high or low from 30 sets of tone combinations. For the duration test, 3 pure tones of $1,000 \mathrm{~Hz}$ lasting either 500 or $250 \mathrm{msec}$ are generated so that the length of 1 tone is different from the other 2. Using these tones, various tonal combinations are created. Subject is asked to identify long or short from 30 sets of tone combinations. For dichotic spondee words test, different words are given to each of the subject's right and left ear, simultaneously. This was carried out in a soundproof booth using bisyllabic words given at most comfortable level. Subjects were asked to identify the 2 words out of 4 choices given on the test sheet. Each test comprises 30 sets of tasks and the score (out of 30) is converted to percentage scores (out of 100).

To assess sentence recognition in the presence of noise, HINT was used [17]. Our main criterion to monitor hearing in noise was the reception threshold for sentence (RTS). RTSs were determined by averaging the presentation levels at which sentences can be recognized $50 \%$ of the time with noise presented at $65 \mathrm{~dB}[18]$. Noise was presented from the front $(0$ degrees azimuth), right and left. Measures in the quiet condition were recorded in $\mathrm{dBA}$ and in the noise conditions, $\mathrm{dB}$ signal to noise ratio. The noise composite score was defined as $(2 \times$ noise front + noise right + noise left $) / 4$. CAP tests and HINTs were evaluated at the beginning of the study and 3, 6, 9, and 12 months after the use of a HA. The participants were tested in the unaided condition.

\section{Ethics}

This study protocol was approved by the Institutional Review Board (2013-0455 and 2016-0574) and registered in the public access site (www.clinicaltrials.gov, NCT01897181). 


\section{Statistics}

The initial evaluation parameters were compared between the HA and the control group using a Mann-Whitney test and a chi-square test, as appropriate. Changes of the evaluation parameters were analyzed using the linear mixed model. Linear mixed regression models were built to estimate the betweengroup differences and the within-group changes (baseline vs. follow-up measures). Standard errors were estimated with the restricted maximum likelihood function, and type III F-tests were preferred. Differences between the groups were examined with planned comparisons at each time point (least square difference tests). The within-group analyses included all five measurements in the time factor. Differences with $p<0.05$ were considered significant. All analyses were conducted in SPSS version 23 (IBM, Armonk, NY, USA).

\section{Results}

\section{Patients}

Sixty-two patients were screened, and 32 participants were enrolled. Six patients discontinued participation within 3 months, and 26 participants (13 in the HA and 13 in the control group) finished the study. The patients chose their HA from 6 different manufacturers, 5 completely-in-the-canal, 7 receiver-in-the-canal, and 1 in-the-ear style HA. Characteristics of patients are summarized in Table 1. There was no significant difference between the two groups regarding age, sex, and duration of discomfort.

All patients showed bilateral moderate hearing loss. SDS was better than $60 \%$ in all patients. The initial hearing levels of the control and HA groups are shown in Table 1. There was no statistical difference between control and HA groups in initial hearing levels, except SDS. SDS of the ear wearing the HA was significantly worse than the SDS of any ears of the control group. The mean thresholds for frequencies of 0.5, 1, 2, and $3 \mathrm{kHz}$ are shown in Fig. 2. The latency of wave $\mathrm{V}$ at a stimulation with $90 \mathrm{~dB}$ HL was $5.79 \pm 0.31 \mathrm{msec}$ in the right and $5.85 \pm 0.40 \mathrm{msec}$ in the left ear of the control group and $5.81 \pm 0.27 \mathrm{msec}$ and $6.00 \pm 0.50 \mathrm{msec}$ in the HA group, respectively. There was no statistical difference in wave $\mathrm{V}$ and I-V latency between the control and HA groups.

The mean aided PTA was $33.82 \pm 7.24 \mathrm{~dB}$ and the mean aided word recognition score was $90.59 \% \pm 10.35 \%$.

\section{Changes of hearing in noise after use of HA}

Estimates and standard errors of HINT scores at initial, 3,

Table 1. Patient characteristics

\begin{tabular}{lccc}
\hline \multicolumn{1}{c}{ Variable } & $\begin{array}{c}\text { Control } \\
(\mathrm{n}=13)\end{array}$ & $\begin{array}{c}\mathrm{HA} \\
(\mathrm{n}=13)\end{array}$ & $\begin{array}{c}\mathrm{p} \text { - } \\
\text { value }\end{array}$ \\
\hline $\begin{array}{l}\text { Age (years) } \\
\text { Sex (male:female) }\end{array}$ & $\begin{array}{c}6.3 \pm 8.0 \\
\text { Initial PTA (dB) }\end{array}$ & $64.9 \pm 7.0$ & 0.29 \\
$\quad$ Right ear & & $5: 8$ & \\
$\quad$ Left ear & $47.8 \pm 14.7$ & $45.6 \pm 8.6$ & 0.32 \\
Initial SD (\%) & $43.7 \pm 6.1$ & $48.0 \pm 8.5$ & 0.07 \\
$\quad$ Right ear & & & \\
$\quad$ Left ear & $94.8 \pm 5.8$ & $90.0 \pm 9.9$ & 0.06 \\
ABR wave V latency (msec) & $94.8 \pm 5.5$ & $84.6 \pm 13.6$ & 0.01 \\
$\quad$ Right ear & & & \\
$\quad$ Left ear & $5.79 \pm 0.31$ & $5.81 \pm 0.27$ & 0.44 \\
ABR wave I-V latency (msec) & $5.85 \pm 0.40$ & $6.00 \pm 0.50$ & 0.23 \\
$\quad$ Right ear & & & \\
Left ear & $4.21 \pm 0.26$ & $4.06 \pm 0.22$ & 0.11 \\
\hline
\end{tabular}

Data are presented as mean \pm standard deviation or number. PTA: pure tone average, SD: speech discrimination, HA: hearing aid, ABR: auditory brainstem response
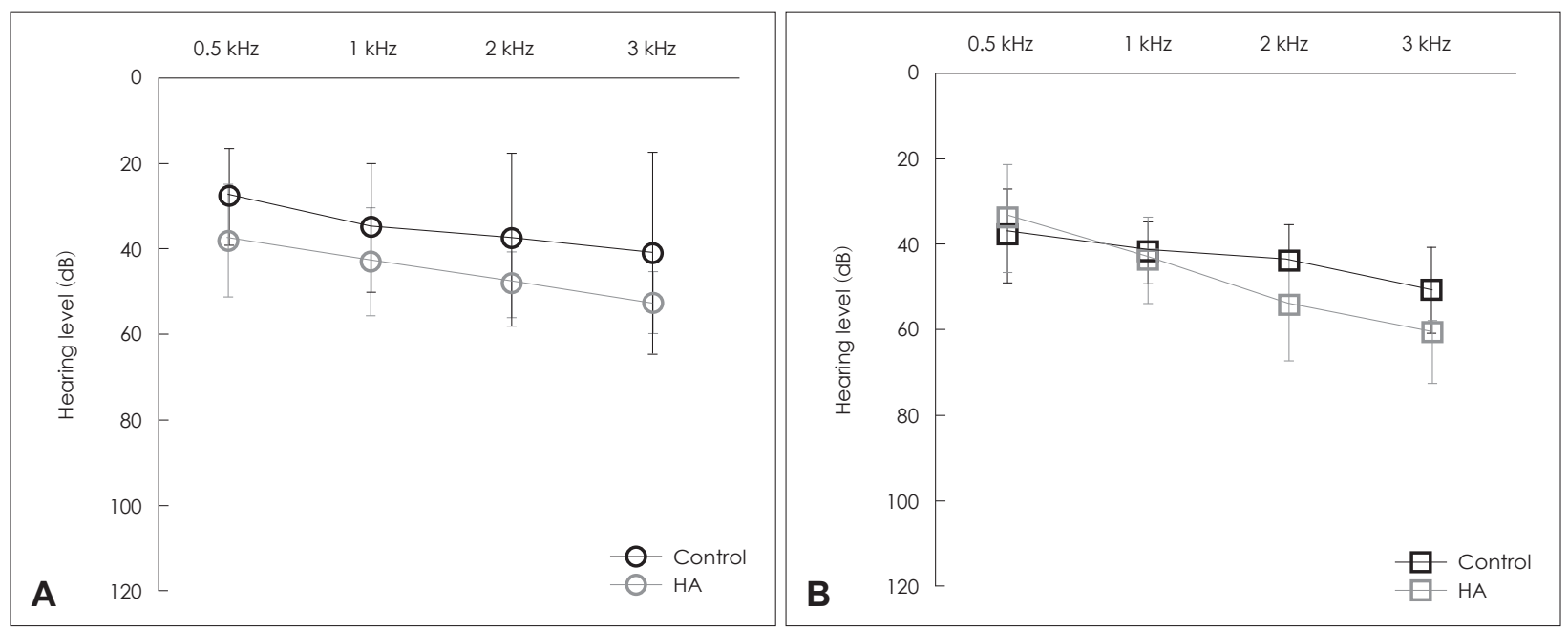

Fig. 2. Mean pure tone thresholds of the right ear $(A)$ and left ear (B). HA: hearing aid. 

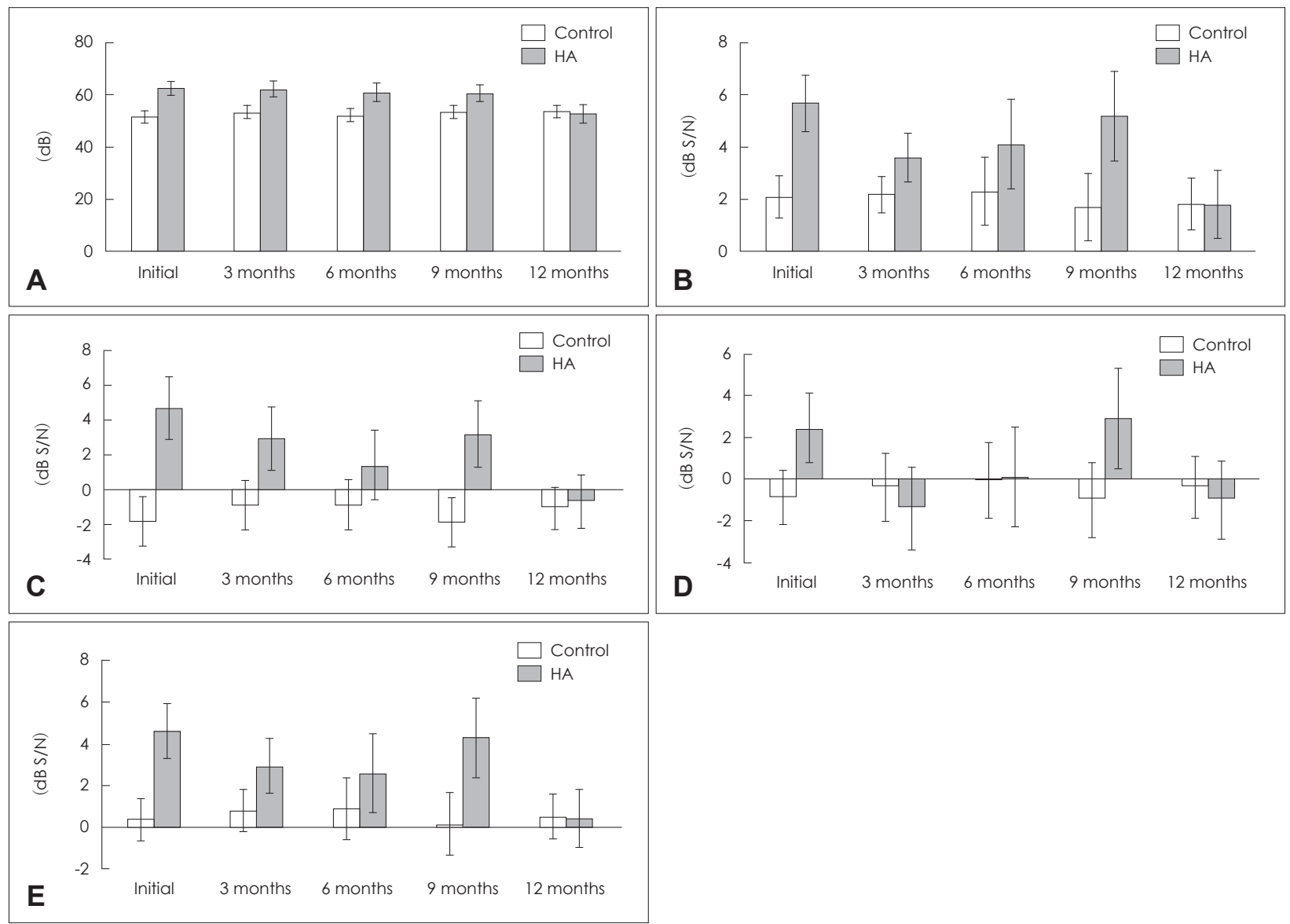

Fig. 3. Estimates and standard errors of hearing in noise test scores at initial, 3,6 , 9, and 12 months calculated by the linear mixed model performed in quiet $(A)$, with noise from front $(B)$, with noise from right $(C)$, with noise from left $(D)$, and composite score (E). HA: hearing aid, $\mathrm{S} / \mathrm{N}$ : signal to noise ratio.

6,9 , and 12 months calculated by the linear mixed model are shown in Fig. 3. The change in HINT scores over time was not different between the HA and control groups. There were significant effects of group with quiet conditions $(p<0.01)$, noise from the front $(p=0.01)$, noise from the right $(p=0.04)$ and the composite score $(p=0.02)$. There was no significant effect of group observed in the noise from the left condition $(p=0.13)$. Additionally, there were no effects of time with regard to all HINT parameters.

\section{Changes of temporal processing after use of HA}

Estimates and standard errors of the frequency, duration, and dichotic tests at initial, 3, 6, 9, and 12 months calculated by the linear mixed model are shown in Fig. 4. There was no significant effect of group observed in the results of the frequency, duration, and dichotic tests. There were significant effects of time regarding the frequency test $(p<0.01)$, and the duration test $(p=0.02)$, but not the dichotic test. Within the HA group, the time point at which the scores of the frequency test started to improve was at 6 months onwards; the scores of the duration test began to improve after 9 months of HA use.

\section{Discussion}

This was a prospective study evaluating changes in hearing in noise abilities and temporal processing after use of a monaural HA in patients with bilateral hearing loss. Our data showed improvements in frequency and duration tests after 1 year of HA use. However, we did not observe changes in hearing in noise abilities or dichotic listening within this period. This suggested that the use of HAs may have some beneficial effect on the participants' auditory performance, even when tested under unamplified conditions.

The improvements in the frequency and duration tests could be attributed to the fact that most of the patients in the HA group had a sloping audiogram with hearing loss in the higher frequencies. Using a HA could have aided them to perform better after amplification of the higher frequencies, especially on the frequency test. Duration and frequency tests are used to evaluate the temporal resolution of signal perception, which 

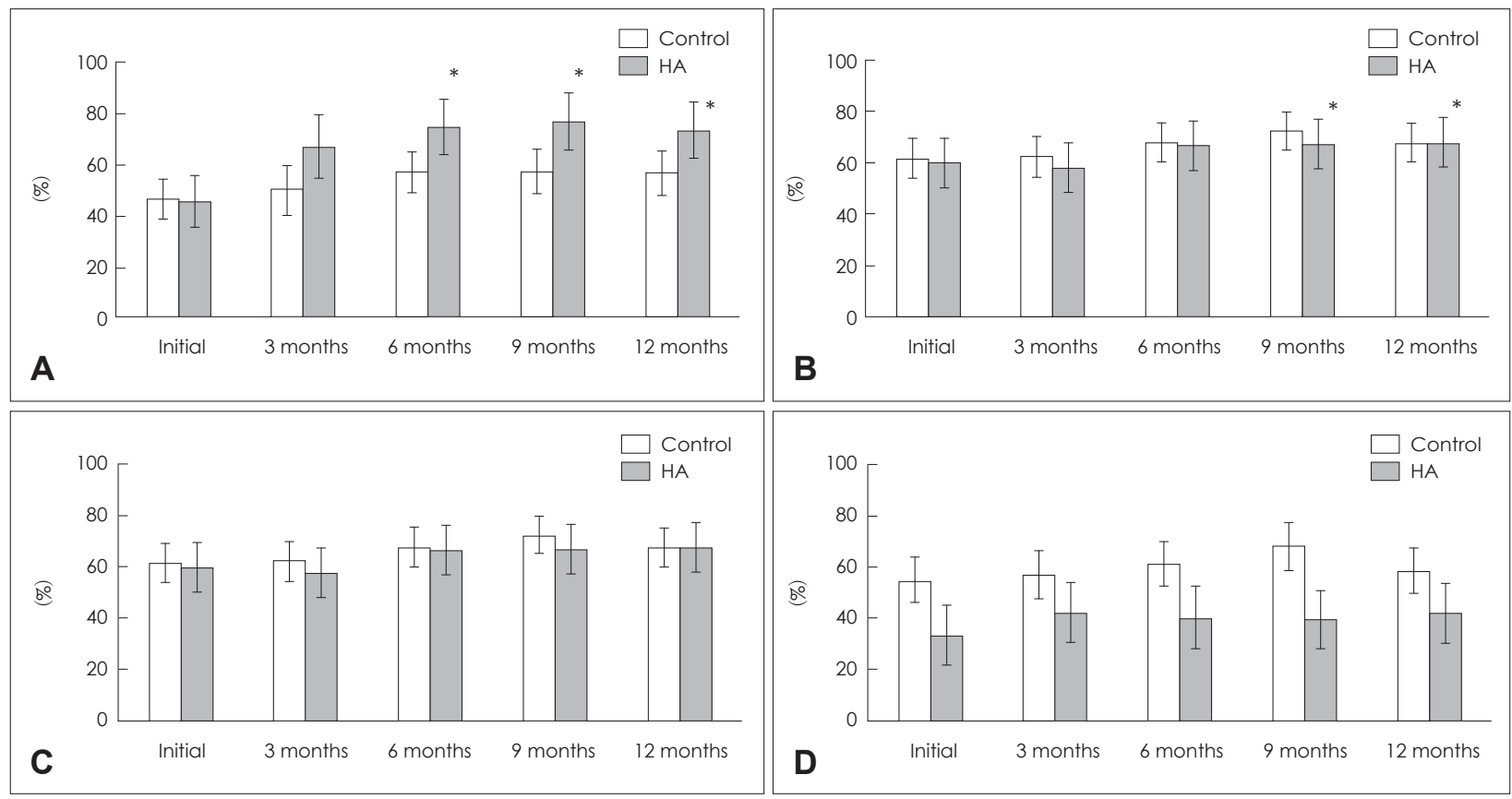

Fig. 4. Estimates and standard errors of the frequency (A), duration (B), right dichotic (C), and left dichotic (D) test scores at initial, 3,6 , 9 , and 12 months calculated by the linear mixed model. *indicates $p<0.05$ for overall time $p$-value. HA: hearing aid.

has been shown to improve with HA use $[19,20]$. The duration pattern test demonstrates good sensitivity $(86 \%)$ and specificity $(92 \%)$ in adults. The frequency pattern test demonstrates $83 \%$ for both sensitivity and specificity [16].

In the current study, the results of the dichotic test did not show improvement over the course of 1 year. This could be because the participants were only fitted with a monaural HA. In the study by Lavie, et al. [21], in which two-thirds of the study participants were fitted sequentially with a one-month term, participants did not show improvements in dichotic scores after 1 month of monaural HA use. However, after being fitted bilaterally, the participants' dichotic scores improved at the 1-month point. The dichotic improvement observed with that study is due to the use of bilateral HAs, which is in line with well-established benefits of bilateral amplification [22].

The speech test in noise scores did not change over the course of 1 year. The ability to hear in a noisy environment is generally considered to be independent of the ability to hear in quiet and exhibits large inter-subject variability [23]. This was also observed in our cohort. Understanding speech in noise depends on the listener's ability to distinguish the target signal from background sounds [24]. Acoustic cues assist this process by providing information for grouping auditory features into objects and identifying a speaker's voice [25]. Hearing in noise also relies upon cognitive and linguistic factors, such as attention, working memory, and lexical knowledge [26]. One suggestion that has been made in the literature is that periodic fine tuning of HAs is important for better fitting and better acclimatization leading to possible improvements in hearing in noise abilities [21]. We did not collect information on how regularly the patients used their HA and how long they used it within their routine daytime activities. With regular tuning and use, better acclimatization could yield different results.

There are some limitations of this study. One limitation of this study was the unequal hearing level between the groups. The control group had better hearing levels than the HA group. A HA was recommended to the patients with a PTA worse than $40 \mathrm{~dB}$ and with SDS better than $60 \%$. Patients who refused to use a HA comprised the control group and patients who agreed to use it comprised the HA group. Because the final decision to use the HA or not was made by the patient, those with worse hearing levels tended to accept the HA. Second, the patients included in the present study were relatively young with only two patients over the age of 70 in the control group and three in the HA group. Both central and peripheral components of the auditory function are attributed to age-related hearing loss [27]. From the 70's onwards, central processing deteriorates at a faster rate than peripheral processing [28]. The variation in patient age could have acted as a bias and further studies consisting of a more uniform age group could lead to different results. Third limitation was that the HA was applied to a unilateral ear. Some reports suggest that unilateral amplification is superior in speech recognition in background noise and that bilateral amplification tends to have a deleterious effect among older patients [29]. However, our cohort comprised a relatively younger group of patients; thus, the ef- 
fectiveness of HAs could be maximized with bilateral application. The test of HA function, however, was evaluated in each ear, and the long-term use of a unilateral HA (1 year) may be enough to show a positive effect of HA use.

In conclusion, the present study demonstrated improvements in frequency and dichotic test scores in patients with bilateral hearing loss after 1 year of HA use. However, we did not observe changes in hearing in noise abilities within this period. These results suggest the possibility of improvement in CAP after the use of a HA. However, this needs to be investigated further in larger and randomized cohorts. Also, changes of hearing in noise abilities and central processing beyond 1 year of HA use could yield different results.

\section{Acknowledgments}

We would like to thank Department of Biostatistics, Asan Medical Center, University of Ulsan College of Medicine, Seoul, Korea for statistical analysis.

This study was supported by a grant (2013-0455) from Asan Institute for Life Sciences, Asan Medical Center, Seoul, Korea.

\section{Conflicts of interest}

The authors have no financial conflicts of interest.

\section{Author Contributions}

Conceptualization: Chan Joo Yang, Myung Hoon Yoo, Chan Il Song, Jong Woo Chung. Data curation: all authors. Funding acquisition: Jong Woo Chung. Investigation: Chan Joo Yang, Myung Hoon Yoo, Chan Il Song. Methodology: Chan Joo Yang, Myung Hoon Yoo, Chan Il Song. Supervision: Jong Woo Chung. Writing — original draft: Yehree Kim. Writing — review \& editing: Jong Woo Chung. Approval of final manuscript: all authors.

\section{ORCID iDs}

Yehree Kim

Chan Joo Yang

Myung Hoon Yoo

Chan Il Song

Jong Woo Chung

https://orcid.org/0000-0002-3056-198X https://orcid.org/0000-0002-9812-5260 https://orcid.org/0000-0002-9158-8924 https://orcid.org/0000-0002-2705-4100 https://orcid.org/0000-0003-0765-9134

\section{REFERENCES}

1) Im GJ, Ahn JH, Lee JH, do Han K, Lee SH, Kim JS, et al. Prevalence of severe-profound hearing loss in South Korea: a nationwide population-based study to analyse a 10-year trend (2006-2015). Sci Rep 2018;8:9940.

2) Stevens G, Flaxman S, Brunskill E, Mascarenhas M, Mathers CD, Finucane M. Global and regional hearing impairment prevalence: an analysis of 42 studies in 29 countries. Eur J Public Health 2013;23: 146-52.

3) Albers MW, Gilmore GC, Kaye J, Murphy C, Wingfield A, Bennett DA, et al. At the interface of sensory and motor dysfunctions and Alzheimer's disease. Alzheimers Dement 2015;11:70-98.

4) Lin FR, Ferrucci L, Metter EJ, An Y, Zonderman AB, Resnick SM. Hearing loss and cognition in the Baltimore Longitudinal Study of Aging. Neuropsychology 2011;25:763-70.

5) Mahmoudi E, Basu T, Langa K, McKee MM, Zazove P, Alexander $\mathrm{N}$, et al. Can hearing aids delay time to diagnosis of dementia, de- pression, or falls in older adults? J Am Geriatr Soc 2019;67:2362-9.

6) McCormack A, Fortnum H. Why do people fitted with hearing aids not wear them? Int J Audiol 2013;52:360-8.

7) Task Force on Central Auditory Processing Consensus Development. Central auditory processing: current status of research and implications for clinical practice. Am J Audiol 1996;5:41-52.

8) Moore BC. The role of temporal fine structure processing in pitch perception, masking, and speech perception for normal-hearing and hearing-impaired people. J Assoc Res Otolaryngol 2008;9:399-406.

9) Song JH, Skoe E, Banai K, Kraus N. Perception of speech in noise: neural correlates. J Cogn Neurosci 2011;23:2268-79.

10) Ziegler JC, Pech-Georgel C, George F, Alario FX, Lorenzi C. Deficits in speech perception predict language learning impairment. Proc Natl Acad Sci U S A 2005;102:14110-5.

11) Lin FR, Albert M. Hearing loss and dementia-who is listening? Aging Ment Health 2014;18:671-3.

12) Lin FR, Yaffe K, Xia J, Xue QL, Harris TB, Purchase-Helzner E, et al. Hearing loss and cognitive decline in older adults. JAMA Intern Med 2013;173:293-9.

13) Tesch-Römer C. Psychological effects of hearing aid use in older adults. J Gerontol B Psychol Sci Soc Sci 1997;52:P127-38.

14) Ahn JH, Lee KS, Yoon TH, Chung JW. The development of test tools for diagnosis of central auditory processing disorder. Korean J Audiol 2005;9:16-22.

15) Musiek FE, Baran JA. Central auditory assessment: thirty years of challenge and change. Ear Hear 1987;8:22S-35S.

16) Musiek FE, Baran JA, Pinheiro ML. Duration pattern recognition in normal subjects and patients with cerebral and cochlear lesions. Audiology 1990;29:304-13.

17) Moon SK, Mun HA, Jung HK, Soli SD, Lee JH, Park K. Development of sentences for Korean hearing in noise test (KHINT). Korean J Otolaryngol-Head Neck Surg 2005;48:724-8.

18) Stuart A. Reception thresholds for sentences in quiet, continuous noise, and interrupted noise in school-age children. J Am Acad Audiol 2008;19:135-46.

19) Mondelli MFCG, Santos MMD, Feniman MR. Unilateral hearing loss: benefit of amplification in sound localization, temporal ordering and resolution. Codas 2019;32:e20180202.

20) Brennan MA, McCreery RW, Buss E, Jesteadt W. The influence of hearing-aid gain on gap-detection thresholds for children and adults with hearing loss. Ear Hear 2018;39:969-79.

21) Lavie L, Banai K, Karni A, Attias J. Hearing aid-induced plasticity in the auditory system of older adults: evidence from speech perception. J Speech Lang Hear Res 2015;58:1601-10.

22) Dillon H. Hearing aids. London: Hodder Arnold;2008.

23) Wilson RH, McArdle R. Speech signals used to evaluate functional status of the auditory system. J Rehabil Res Dev 2005;42:79-94.

24) Thompson EC, Woodruff Carr K, White-Schwoch T, Otto-Meyer S, Kraus N. Individual differences in speech-in-noise perception parallel neural speech processing and attention in preschoolers. Hear Res 2017;344:148-57.

25) Shamma SA, Elhilali M, Micheyl C. Temporal coherence and attention in auditory scene analysis. Trends Neurosci 2011;34:114-23.

26) Lewis D, Hoover B, Choi S, Stelmachowicz P. Relationship between speech perception in noise and phonological awareness skills for children with normal hearing. Ear Hear 2010;31:761-8.

27) Gates GA. Central auditory processing in presbycusis: an epidemiologic perspective. In: Hickson L, editor. Hearing care for adults 2009 - The challenge of aging. Proceedings of the Second International Adult Conference; 2009 Nov 16-18: Chicago, Illinois. Staefa: Phonak AG;2009. p.47-52.

28) Gates GA, Feeney MP, Mills D. Cross-sectional age-changes of hearing in the elderly. Ear Hear 2008;29:865-74.

29) Walden TC, Walden BE. Unilateral versus bilateral amplification for adults with impaired hearing. J Am Acad Audiol 2005;16:574-84. 